\title{
Observation of a high-quality quasi-periodic rapidly propagating wave train using SDO/AIA ${ }^{\star}$
}

\author{
G. Nisticò ${ }^{1}$, D. J. Pascoe ${ }^{1}$, and V. M. Nakariakov ${ }^{1,2,3}$ \\ ${ }^{1}$ Centre for Fusion, Space and Astrophysics, Department of Physics, University of Warwick, Coventry CV4 7AL, UK \\ e-mail: g.nistico@warwick.ac.uk \\ 2 School of Space Research, Kyung Hee University, 446-701 Yongin, Gyeonggi, Korea \\ 3 Central Astronomical Observatory at Pulkovo of the Russian Academy of Sciences, 196140 St Petersburg, Russia
}

Received 5 March 2014 / Accepted 18 June 2014

\begin{abstract}
Context. We present a new event of quasi-periodic wave trains observed in EUV wavebands that rapidly propagate away from an active region after a flare.

Aims. We measured the parameters of a wave train observed on 7 December 2013 after an M1.2 flare, such as the phase speeds, periods and wavelengths, in relationship to the local coronal environment and the energy sources.

Methods. We compared our observations with a numerical simulation of fast magnetoacoustic waves that undergo dispersive evolution and leakage in a coronal loop embedded in a potential magnetic field.

Results. The wave train is observed to propagate as several arc-shaped intensity disturbances for almost half an hour, with a speed greater than $1000 \mathrm{~km} \mathrm{~s}^{-1}$ and a period of about $1 \mathrm{~min}$. The wave train followed two different patterns of propagation, in accordance with the magnetic structure of the active region. The oscillatory signal is found to be of high-quality, i.e. there is a large number (10 or more) of subsequent wave fronts observed. The observations are found to be consistent with the numerical simulation of a fast wave train generated by a localised impulsive energy release.

Conclusions. Transverse structuring in the corona can efficiently create and guide high-quality quasi-periodic propagating fast wave trains.
\end{abstract}

Key words. Magnetohydrodynamics (MHD) - waves - Sun: corona - Sun: oscillations - methods: numerical methods: observational

\section{Introduction}

Observations of the Sun at the extreme ultraviolet (EUV) and X-ray wavelengths by space-borne instruments in the past twenty years allowed us to clearly detect several magnetohydrodynamic (MHD) wave modes that propagate through the solar corona, which confirmed previously formulated theories and models (Zajtsev \& Stepanov 1975; Roberts 1981a,b; Edwin \& Roberts 1983). MHD wave activity has been observed mainly as damped transverse (kink) oscillations of coronal loops (De Moortel \& Nakariakov 2012) and decayless kink oscillations (Nisticò et al. 2013; Anfinogentov et al. 2013), as well as global coronal "EIT" or "tsunami" waves (Patsourakos et al. 2009), standing and propagating slow magnetoacoustic waves (Wang 2011; De Moortel 2009), and sausage oscillations in radio wavebands (Nakariakov \& Melnikov 2009). Recently, the high-time resolution and sensitivity of the Atmospheric Imaging Assembly (AIA) onboard the Solar Dynamics Observatory (SDO) allowed Liu et al. $(2011,2012)$ to detect rapidly propagating wave trains of the EUV emission intensity during flare/CME events. The observed disturbances were interpreted in terms of fast magnetoacoustic waves driven quasi-periodically at the base of the flaring region (Ofman et al. 2011). Other detailed observations are reported by Shen \& Liu (2012) and Shen et al. (2013), who demonstrated an intimate association with the flare

$\star$ The movies are available in electronic form at http: //www . aanda.org energy releases and the role of magnetic loop structures in guiding these waves. Yuan et al. (2013) also observed quasiperiodic propagating fast wave trains associated with flaring radio emission. Rapidly propagating waves of the EUV emission intensity disturbances should not be confused with the wellunderstood and analogous slow waves (see, e.g. De Moortel 2009). These waves also form wave trains (e.g. Sych et al. 2012), but propagate along the apparent magnetic field lines at essentially sub-sonic speeds, and their periods are typically longer than 120 s. Pascoe et al. (2013) developed an advanced numerical model for the dispersive evolution of fast MHD waves in an expanding magnetic field generated by an impulsive, spatially localised energy release with a field-aligned density structure which produced results consistent with the observations of Yuan et al. (2013).

Originally, dispersive evolution of broadband fast sausage waves was proposed by Roberts et al. (1984) as a mechanism for creating quasi-periodic fast wave trains such as those detected in radio emission from solar flares (Roberts et al. 1983). The predicted behaviour was confirmed by initial numerical studies (e.g. Murawski \& Roberts 1993a,b). For the developed stage of the evolution the quasi-periodic wave trains are best described using wavelet analysis (Nakariakov et al. 2004), which reveals the "crazy tadpole" characteristic signature also seen in data for propagating fast wave trains detected with the Solar Eclipse Coronal Imaging System (SECIS; Katsiyannis et al. 2003; Cooper et al. 2003) and in decimetric band radio bursts 
(e.g. Mészárosová et al. 2009a,b, 2011, 2013; Karlický et al. 2011, 2013). Recent numerical studies have extended models for dispersive evolution of impulsively generated wave trains to include current sheets (Jelínek \& Karlický 2012; Jelínek et al. 2012).

Despite the growing interest in rapidly propagating EUV wave trains in solar coronal structures, their observational detection is rare, with just a few events published in literature (Liu et al. 2011; Shen \& Liu 2012; Shen et al. 2013). Thus, any new observation of this phenomenon is very valuable to reveal its nature, validate and further develop the theoretical models. Here, we report the observation of a quasi-periodic fast magnetoacoustic propagating (QFP) wave train with a high signal quality, that is, with a large number (10 or more) of wave fronts. The observed event is described in Sect. 2. In Sect. 3 we present a numerical model for fast wave trains based on dispersive evolution within a coronal loop embedded in an active region. A detailed discussion and comparison of our observational and numerical results are given in Sect. 4.

\section{Observations}

A flare event of class M1.2 was registered by the SDO/AIA telescopes between 07:00-08:00 UT on 7 December 2013 in the active region (AR) NOAA 11909 (S17 W62). The event is listed in the Last events catalogue of the SolarSoft $\operatorname{archive}^{1}$, and a preview of the animation revealed a wave train propagating at $171 \AA$. The flare triggered a fast-propagating coronal mass ejection (CME) with an average speed of $909 \mathrm{~km} \mathrm{~s}^{-1}$, as recorded in the Computer Aided CME Tracking (CACTus) catalog ${ }^{2}$. We downloaded full-cadence FITS files from SolarSoft (SSW) using the function vso_search.pro in the time range as indicated previously and collected a total of 300 frames. The data were prepped and corrected using the standard routine aia_prep.pro, normalised to the exposure time, since this changes in response to the increase of the EUV emission due to the flare, ranging between $1.13 \mathrm{~s}$ and $1.99 \mathrm{~s}$ (the latter value is the standard exposure time for the 171 filter). We extracted a field of view $(\mathrm{FOV})$ of $1000 \times 1000 \mathrm{px}$ from the full-size images (equivalent to $600^{\prime \prime} \times 600^{\prime \prime}$ ), which encloses the region of interest (ROI), with the bottom-left corner at $\left(4522^{\prime \prime} 10,-6277^{\prime} 90\right)$ and the top-right corner at (1051'.50, -28.'50).

Figure 1 shows a potential field source surface (PFSS) model for the solar magnetic field at the time 06:04:32, just one hour before our observation. The magnetic field model was downloaded from the PFSS dataset ${ }^{3}$ and processed with the standard PFSS routines available in the SSW package ${ }^{4}$.

To highlight the propagation of the wave train, we constructed running-difference images (RDIs) by subtracting each image from the next one, therefore with a $\Delta t$ of $12 \mathrm{~s}$ corresponding to the AIA-171 cadence. The difference operation creates a salt-and-pepper noise effect in the new images, which we removed by smoothing each RDI with a 2D Gaussian kernel. In IDL we used the gauss_smooth function with a $\sigma=1.5$.

\footnotetext{
1 http://wwW. lmsal.com/solarsoft/

latest_events_archive.html

2 http://sidc.oma.be/cactus/catalog/LASC0/2_5_0/qkl/

2013/12/CMEQ028/CME.html

3 http://www. lmsal.com/solarsoft/pfss/kitrun48/

Bfield-bydate/

4 see http://www. Imsal. com/ derosa/pfsspack/ for a tutorial.
}

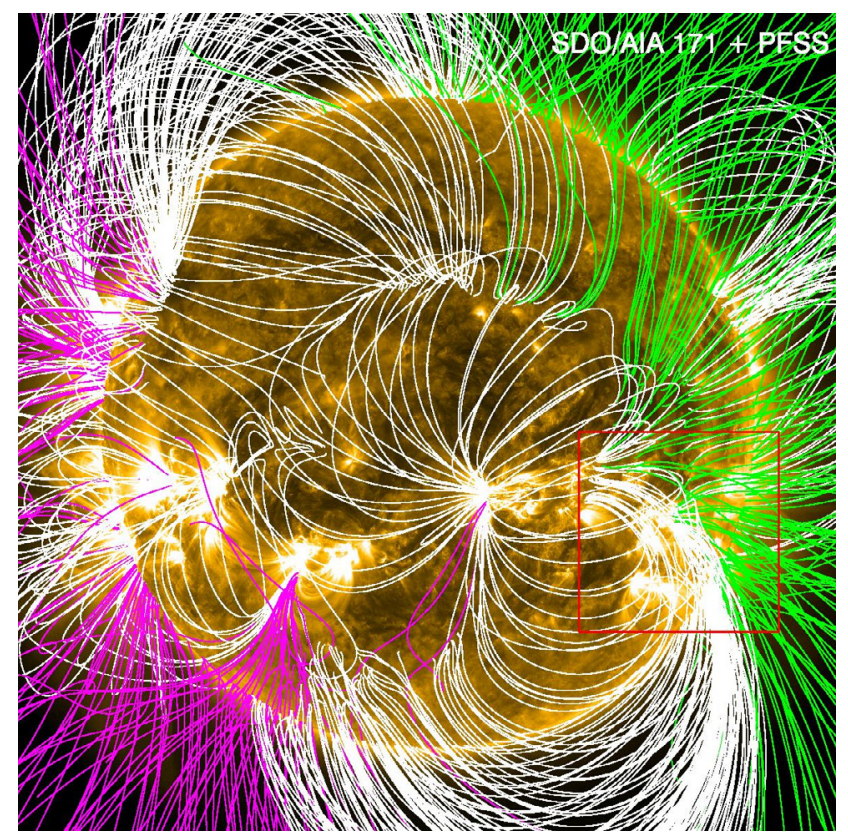

Fig. 1. PFSS model for the solar magnetic field during our observations (ROI indicated by the red box). The white field lines are closed, while the green and purple represent open field lines with opposite polarities.

Figure 2 provides a time sequence of the development and evolution of the wave train. We summarise the different phases step by step:

- 07:06-07:18 UT, some loops are progressively expanding in the active region (panel $b$ ).

- 07:20-07:24 UT, diffraction pattern visible in $171 \AA$ A images due to the flare emission and appearance of an almost circular front, associated with the erupting $\mathrm{CME} /$ shock (panel c).

- 07:25:35 UT, appearance of a first front of the narrow wave train along a curved path (panel d).

- 07:26-07:37 UT, development of the large extended wave train, propagating away from the flare region. Several distinct fronts are visible (panels e-f).

- 07:39-08:00 UT, formation of post-flare loops. At about 07:47-07:50 UT some wave train fronts are seen to propagate off-limb (panel h).

A movie showing the temporal evolution of the wave train is available online.

\subsection{Slit analysis}

We constructed time-distance (TD) maps to estimate the main physical parameters of the wave train from the filtered RDIs, such as the average speed and period. We considered two slits: a curved path, oriented along the narrow wave train (red line in Fig. 2), and a straight line following the large wave train (green line in Fig. 2). The intensity profile, averaged over a width of 21 pixels, is recovered along the slit for each frame, and stacked progressively in time. Furthermore, TD maps from the RDIs were again filtered by a Gaussian smoothing operator to improve contrast and attenuate the intensity steepness between pixels caused by the stacking operation or by image-diffraction patterns due to the flare emission.

The TD maps are shown along with the temporal evolution of soft X-rays from the GOES-15 satellite in Fig. 3. The red dots in 


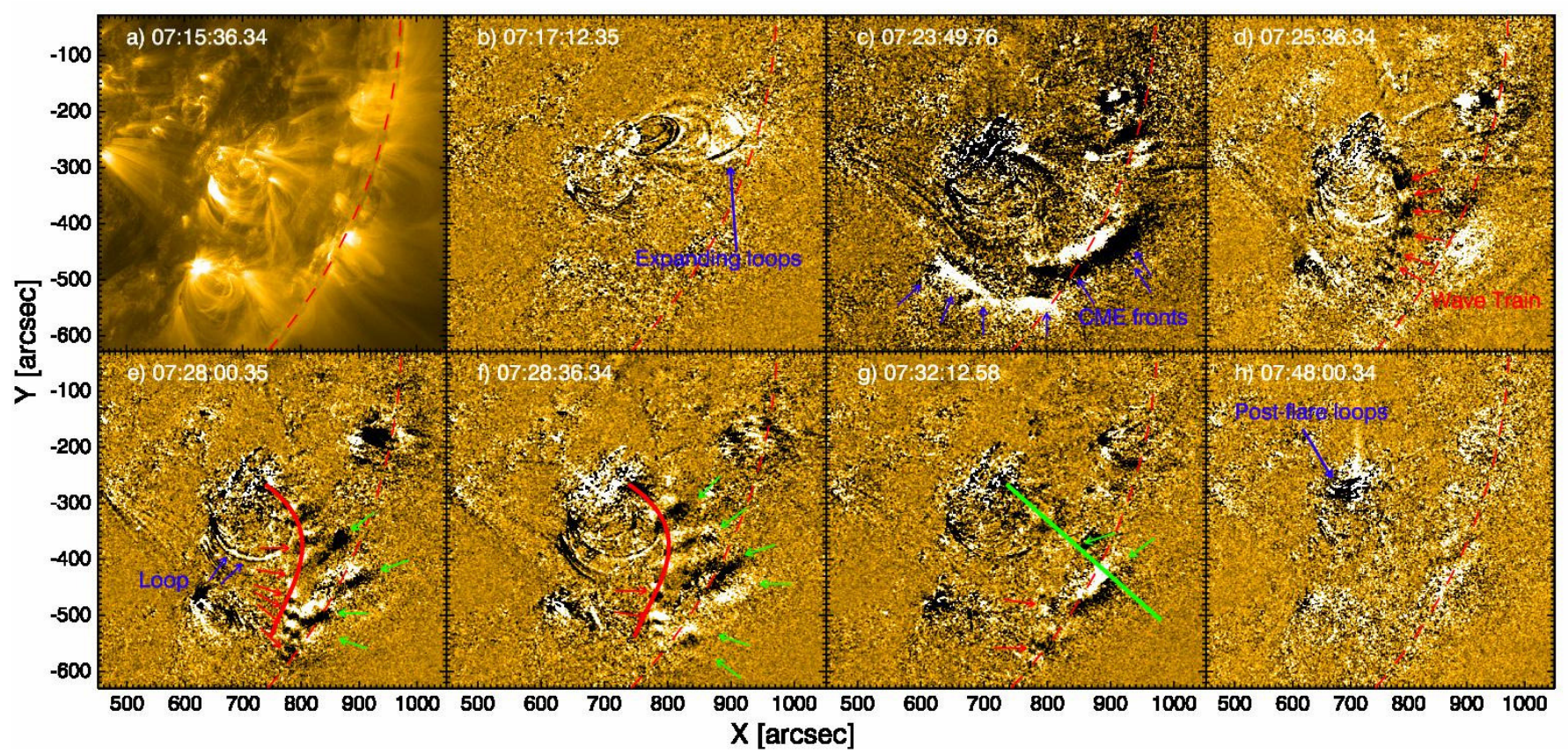

Fig. 2. Sequence of the SDO/AIA FOV at $171 \AA$ showing the formation of the wave train. Panel a) shows the analysed FOV in normal intensity, other panels show it in running-difference intensity at different instants of time to highlight dynamical changes. We outline with blue arrows some temporarily evolving features, such as expanding loops b), CME fronts c), oscillating loops during the flare e), post-flare loops $\mathbf{h}$ ). The wave train fronts are visible in panels d)-g) as periodically spaced bright and dark regions. The red arrows mark the position of a narrow wave train that follows a curved path, while the green arrows mark a large extended wave train. The red and green lines in panels e)-f) and g) locate the position of slits to create time-distance maps. A movie with the temporal evolution is available online, with the left panel showing the normal-intensity images and the right one the running-difference images.

the TD maps locate the positions of the shock/CME fronts as determined by inspection. The green and blue lines are the curves of best fit (see parameters listed in Table 1). The vertical dashed red lines mark the position of the start and peak of flare activity, which agree well with the initial and final exponential growth of the eruption front. The expanding bubble that gives origin to the CME detected with coronagraphs slowly expands with a constant speed of the order of $10 \mathrm{~km} \mathrm{~s}^{-1}$, and then experiences a rapid acceleration in almost $20 \mathrm{~s}$.

The QFP wave trains are depicted by periodic straight intensity variations after the outburst and last for several minutes. The first TD map corresponds to the QFP wave train that propagates for almost $15 \mathrm{~min}$ along a curved path in a narrow region, probably the boundary of a coronal funnel with a closed field loop; the second map shows the large extended wave train propagating as arc-shape fronts and lasting for about $35 \mathrm{~min}$. The average speed of the wave train fronts is about $1 \mathrm{Mm} \mathrm{s}^{-1}$ and the period is between 60-80 s. More details are given in Sect. 4.

The underlying coronal region has a complex magnetic topology, characterised by closed magnetic field lines and an extended magnetic funnel, that is also shown in the PFSS model. In the next section we present a numerical model based on these observations.

\section{Numerical modelling}

In this section we compare our observation of high-quality propagating wave trains with a numerical model of fast MHD waves that undergo dispersive evolution in a structured active region. Pascoe et al. (2013) studied the dispersive evolution of fast MHD waves in an expanding magnetic field with a field-aligned density structure. To adapt our modelling to the current observation, we extended the model of Pascoe et al. (2013) to a curved coronal loop filled in with enhanced plasma density. The gravitational force is not included in the model.

Fast magnetoacoustic waves are excited by an impulsive compressive perturbation near one of the footpoints of the loop. The initial perturbation has a sausage-mode symmetry. The dense loop acts as a waveguide for fast magnetoacoustic waves and the transverse length scale causes geometrical dispersion, which leads to the formation and evolution of quasiperiodic wave trains. The leaky nature of the sausage mode allows wave train components with a sufficiently long wavelength to radiate away from the coronal loop and propagate through the open corona (Nakariakov et al. 2012).

The simulation was performed using LARE2D (Arber et al. 2001). This code solves the ideal MHD equations by taking a Lagrangian predictor-corrector time step, after which variables are remapped back onto the original Eulerian grid using van Leer gradient limiters. The $2.5 \mathrm{D}$ approximation employed corresponds to no gradients in the $z$ direction, i.e. $\partial / \partial z=0$. The numerical domain is composed of $4000 \times 2000$ grid points, which convergence tests demonstrate is sufficient to accurately resolve the problem. The boundary conditions are periodic in the horizontal direction and line-tied in the vertical direction. This allows reflections to occur at the base of the corona (e.g. at loop footpoints), but those from the boundary at the upper corona are not desired, and the simulation ends before they affect the results.

In normalised units, the numerical domain has a size $2 \pi \times \pi$ and the runtime is $\tilde{t}=4$. We converted this to physical units by choosing appropriate normalisation constants for speed $\left(v_{0}\right)$ and time $\left(t_{0}\right)$, with the normalisation length scale then given by $l_{0}=$ $v_{0} t_{0}$. The physical length scales $(x, y)$, time scales $(t)$, and speeds $(v)$ are related to the dimensionless variables (denoted by tildes) by $x=\tilde{x} l_{0}, y=\tilde{y} l_{0}, t=\tilde{t} t_{0}$, and $v=\tilde{v} v_{0}$. Our normalisation 

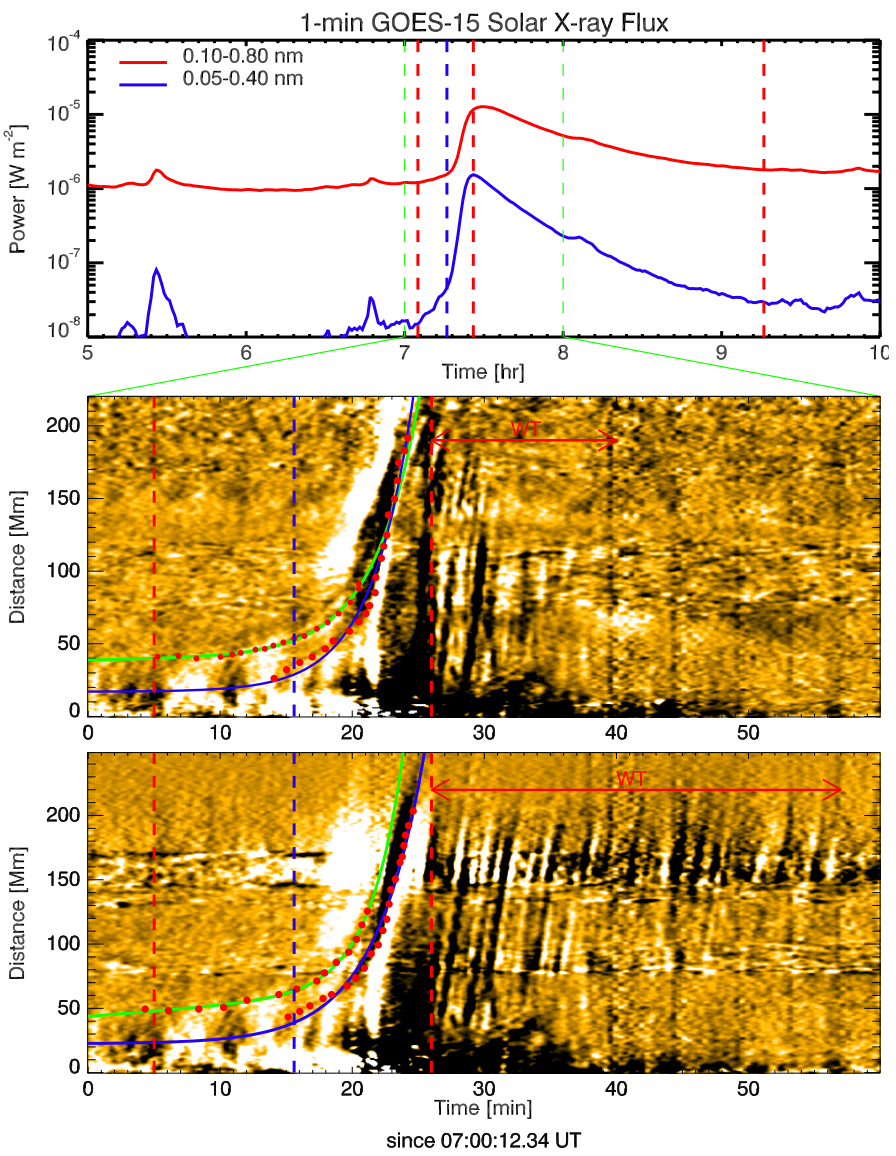

Fig. 3. Top: temporal evolution of the soft X-ray intensity at short (red) and long (blue) wavelengths from GOES-15. The vertical green dashed lines define the time window of our observation between 07:0008:00 UT. The vertical red dashed lines represent the start, the peak and the end of the flare activity (note the long tail after the peak), whilst the blue dashed line marks the start of the ramp in X-ray flux. Middle and bottom: TD maps from the curved and straight slits. The red dots mark the position in time of the eruption fronts (shock/CME), which are fitted by exponential curves (green and blue lines). Note the correspondence between the initial and exponential growth with the start and peak of the flare (red dashed lines). The wave train is depicted as periodic straight intensity variations (double arrow labelled "WT").

is based on the observational findings presented in Sect. 2. We chose $v_{0}=1 \mathrm{Mm} \mathrm{s}^{-1}$ and $t_{0}=80 \mathrm{~s}$. This gives $l_{0}=80 \mathrm{Mm}$, and therefore the numerical domain has a height of $\approx 250 \mathrm{Mm}$.

The magnetic field used in our model is based on the potential field used by Pascoe et al. (2013), but with a horizontal shift of $\pi / 2$, which places the closed field lines at the centre of the numerical domain rather than the open ones,

$\boldsymbol{B}=B_{0} \exp (-y)\left[\cos (x) \overline{\boldsymbol{e}}_{x}-\sin (x) \overline{\boldsymbol{e}}_{y}\right]$,

where $B_{0}$ is a constant determining the magnitude of the field. As in Fig. 1, the magnetic field is composed of closed field lines with open field lines of opposite polarity on the either sides (see Fig. 4). The density profile for the field-aligned coronal loop is based on the generalised symmetric Epstein profile (e.g. Nakariakov \& Roberts 1995; Pascoe et al. 2007) with additional modifications to take into account the loop curvature and expansion (the increase in width at the apex). The density profile is also stratified in the vertical direction with a scale height $\Lambda$,

$\rho=\left[\left(\rho_{0}-\rho_{\infty}\right) \operatorname{sech}^{2}\left(\frac{r}{a(s)}\right)^{p}+\rho_{\infty}\right] \exp \left(-\frac{y+\pi}{\Lambda}\right)$,
Table 1. Fitting parameters for the CME and wave train fronts.

\begin{tabular}{|c|c|c|c|c|}
\hline \multicolumn{5}{|c|}{ TD MAP 1} \\
\hline \multicolumn{5}{|c|}{ CME front displacement $\left(d=d_{0}+v t+a \exp (b t)\right)$} \\
\hline Front & $v\left[\mathrm{~km} \mathrm{~s}^{-1}\right]$ & $\Delta v\left[\mathrm{~km} \mathrm{~s}^{-1}\right]$ & $a[\mathrm{~km}]$ & $b\left[\mathrm{~s}^{-1}\right]$ \\
\hline 1 & 2.68 & 9.25 & 119 & 17 \\
\hline 2 & 0.00 & 0.00 & 87 & 19 \\
\hline \multicolumn{5}{|c|}{ Wave front displacement $\left(d=d_{0}+v t\right)$} \\
\hline Front & $v\left[\mathrm{~km} \mathrm{~s}^{-1}\right]$ & $\Delta v\left[\mathrm{~km} \mathrm{~s}^{-1}\right]$ & $P[\min ]$ & $L[\mathrm{Mm}]$ \\
\hline 1 & 538 & 126 & - & - \\
\hline 2 & 610 & 48 & 1.24 & 46 \\
\hline 3 & 762 & 170 & 0.83 & 38 \\
\hline 4 & 1119 & 77 & 1.85 & 57 \\
\hline 5 & 1333 & 90 & 0.95 & 76 \\
\hline 6 & 1565 & 236 & 1.13 & 106 \\
\hline 7 & 1288 & 141 & 1.07 & 82 \\
\hline 8 & 1741 & 599 & 0.93 & 97 \\
\hline 9 & 2394 & 1742 & 2.98 & 429 \\
\hline 10 & 923 & 214 & 2.15 & 119 \\
\hline \multicolumn{5}{|c|}{ TD MAP 2} \\
\hline \multicolumn{5}{|c|}{ CME front displacement $\left(d=d_{0}+v t+a \exp (b t)\right)$} \\
\hline Front & $v\left[\mathrm{~km} \mathrm{~s}^{-1}\right]$ & $\Delta v\left[\mathrm{~km} \mathrm{~s}^{-1}\right]$ & $a[\mathrm{~km}]$ & $b\left[\mathrm{~s}^{-1}\right]$ \\
\hline 1 & 13.69 & 5.69 & 15 & 24 \\
\hline 2 & 0.00 & 0.00 & 258 & 16 \\
\hline \multicolumn{5}{|c|}{ Wave front displacement $\left(d=d_{0}+v t\right)$} \\
\hline Front & $v\left[\mathrm{~km} \mathrm{~s}^{-1}\right]$ & $\Delta v\left[\mathrm{~km} \mathrm{~s}^{-1}\right]$ & $P[\min ]$ & $L[\mathrm{Mm}]$ \\
\hline 1 & 1124 & 59 & - & - \\
\hline 2 & 1061 & 48 & 1.14 & 72 \\
\hline 3 & 1265 & 151 & 0.81 & 62 \\
\hline 4 & 1094 & 58 & 1.14 & 75 \\
\hline 5 & 1074 & 56 & 1.06 & 69 \\
\hline 6 & 1144 & 188 & 1.36 & 93 \\
\hline 7 & 1093 & 465 & 1.07 & 70 \\
\hline 8 & 967 & 65 & 1.16 & 67 \\
\hline 9 & 1090 & 128 & 1.47 & 96 \\
\hline 10 & 1210 & 134 & 0.87 & 63 \\
\hline 11 & 1252 & 131 & 1.48 & 111 \\
\hline 12 & 1386 & 317 & 2.18 & 181 \\
\hline 13 & 1400 & 193 & 0.96 & 80 \\
\hline 14 & 1851 & 494 & 0.86 & 96 \\
\hline 15 & 1444 & 215 & 1.65 & 143 \\
\hline 16 & 941 & 82 & 1.46 & 82 \\
\hline 17 & 1145 & 263 & 1.24 & 85 \\
\hline
\end{tabular}

Notes. We list the speed with the associated error, the period, and the wavelength $L$, determined as $L=v P$.

where $r$ is the distance from the loop axis (along the local equipotential line), $a(s)$ is the local loop semi-width (a function of the distance along the loop axis $s=[0, L]), \rho_{0} / \rho_{\infty}$ is the loop density contrast ratio, and the index $p$ determines the profile steepness.

For our simulation we took $\rho_{0} / \rho_{\infty}=5$ and $p=8$. The apex of the loop is located at $(0,-\pi / 2)$, and at this point the loop has a maximum width of $2 a(s=L / 2)=0.36$. The smallest loop width is $2 a(s=0, L)=0.0065$ at the loop footpoints and the loop length (along the axis) is $L=4.51$. Vertical stratification of the Alfvén speed would cause the wavelength of the propagating wave trains to vary as a function of height. Since the FOV of our observations is insufficient to determine any such variation, we chose $\Lambda=0.5$ for simplicity, which means that the coronal loop structure provides the only variation in Alfvén speed. Figure 4 shows the equilibrium used in the numerical simulation. The top panel shows the density (colour contour) and magnetic field lines (Eq. (1)). The coronal loop reduces the local Alfvén speed $C_{\mathrm{A}}$ (bottom panel) from the background value of $1 \mathrm{Mm} \mathrm{s}^{-1}$ and so 
G. Nisticò et al.: Observation of a high-quality quasi-periodic rapidly propagating wave train using SDO/AIA
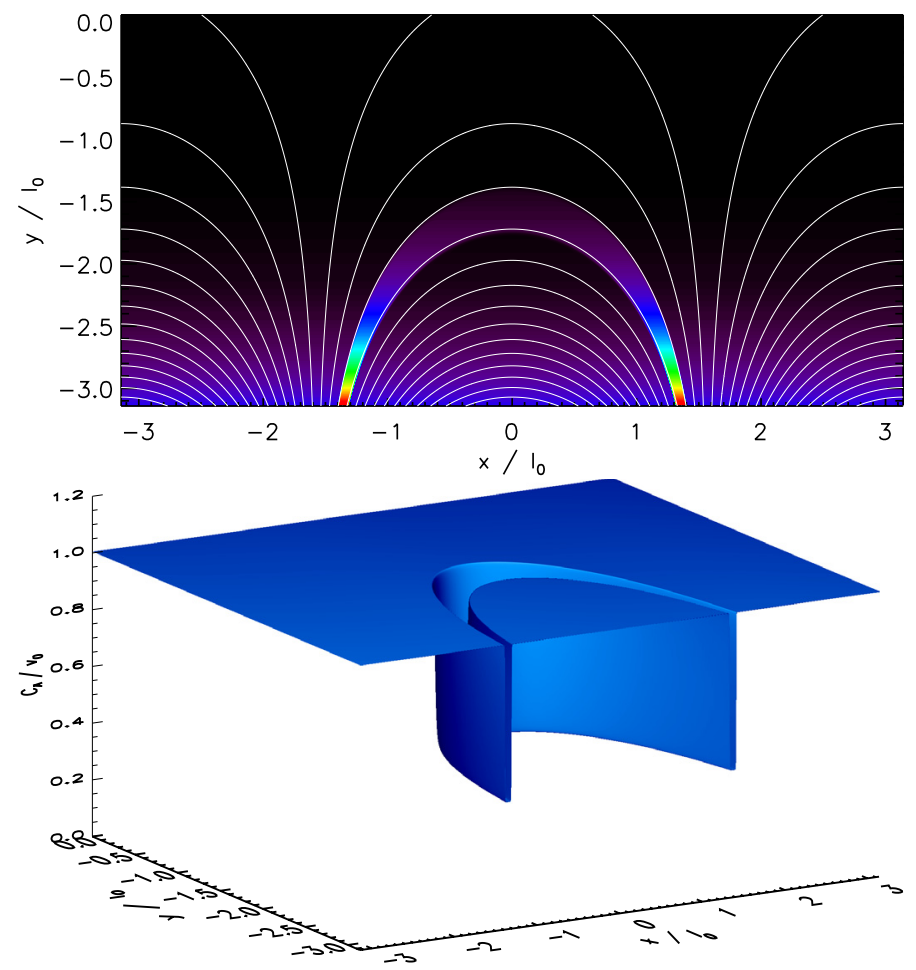

Fig. 4. Equilibrium profiles used for our numerical simulation of a fast wave train generated in an active region. The top panel shows the density (colour contour) and magnetic field lines. The coronal loop has a density contrast ratio $\rho_{0} / \rho_{\infty}=5$ and reduces the local Alfvén speed (bottom panel).

acts as a waveguide for fast magnetoacoustic waves. The highdensity loop is set up to be in equilibrium by defining a temperature profile that satisfies the constant total pressure condition. The plasma $\beta$ is sufficiently small $\left(\beta<10^{-5}\right)$ that we do not consider slow magnetoacoustic waves in this study.

A compressive perturbation was applied near one of the loop footpoints,

$V_{x}=A x \exp \left[-\left(\frac{x-x_{0}}{\Delta_{x}}\right)^{2}\right] \exp \left[-\left(\frac{y-y_{0}}{\Delta_{y}}\right)^{2}\right]$,

where $A$ is the initial (small) amplitude of the perturbation located at $\left(x_{0}, y_{0}\right)=(1.3,-2.9)$ (see Fig. 4-top) and the parameters $\Delta_{x}$ and $\Delta_{y}$ are the width of the initial perturbation in the horizontal and vertical directions, respectively. This perturbation represents a single, spatially localised disturbance such as caused by a solar flare. We chose $\Delta_{x}=\Delta_{y}=0.05$ so that the perturbation is comparable in size to the loop at the location where it is applied. This ensures efficient generation and dispersion of fast sausage waves.

Figure 5 shows the evolution of the compressive perturbation in the numerical simulation. Note that the numerical domain has been rotated for better comparison with the observational data in Fig. 2. The panels show images for the perturbation speed of the plasma at several consecutive instants of time. It is clear that the model generates a fast wave train propagating away from the location of the initial impulsive perturbation. The wave train is clearly quasi-periodic with a high signal quality. It well resembles the observed rapidly propagating quasi-periodic wave trains of the EUV intensity perturbations discussed in Sect. 2. Figure 6 shows the spatial structure of the density perturbations in the excited fast wave train. The impulsive driver in our simulation generates fast sausage oscillations with a broad range
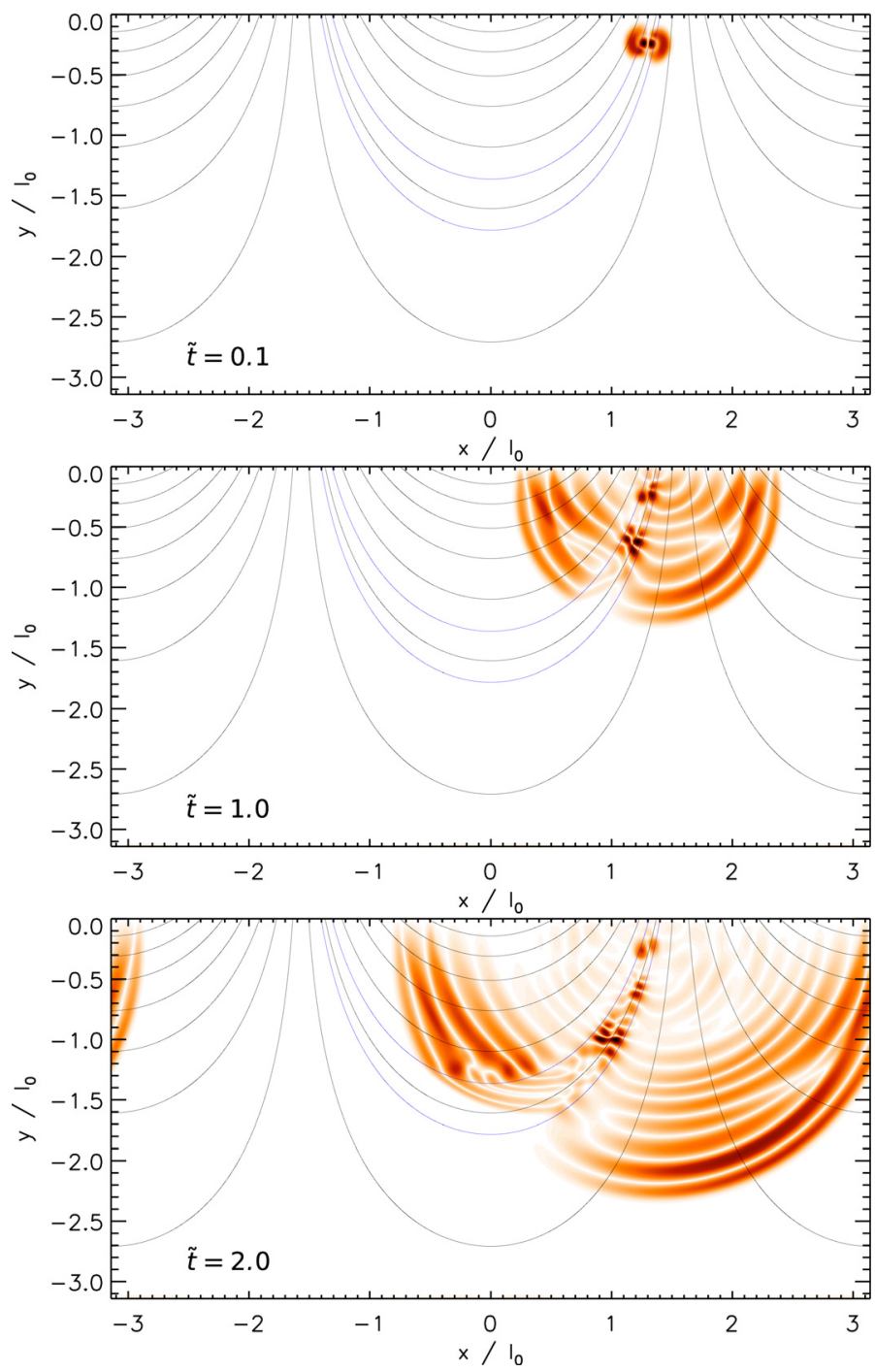

Fig. 5. Snapshots representing the temporal evolution of the absolute value of the induced flow velocity in the numerical simulation, at times $\tilde{t}=0.1,1$ and 2 (from top to bottom). The blue lines outline the wave guiding coronal loop. Note the rotation of the domain in comparison with the top panel in Fig. 4.

of wave numbers. A wave train is generated within the coronal loop, which undergoes dispersive evolution. The components of this wave train that have a wave number greater than the sausage mode cut-off remain trapped in the waveguide and propagate along the loop, whilst those with a sufficiently small wave number gradually leak out of the loop and form the outwardpropagating fast wave trains in the external medium.

The trapped wave train propagates in both directions along the loop axis. For the downward propagating part (i.e. towards the nearest footpoint) the transverse length scale (local loop width) decreases, and therefore the corresponding sausage mode cut-off wave number increases. Wave train components that were previously trapped become leaky and so radiate away from the coronal loop. The trapped wave train acts as a moving source of outward-propagating (leaky) waves and the impulsively deposited energy is released from the loop over an extended time. In this way, a propagating quasi-periodic wave train with a high signal quality can be generated from a single initial perturbation.

Figure 7 shows fast wave trains at two locations, inside and outside the wave-guiding loop (see Fig. 6). The quasi-periodic 


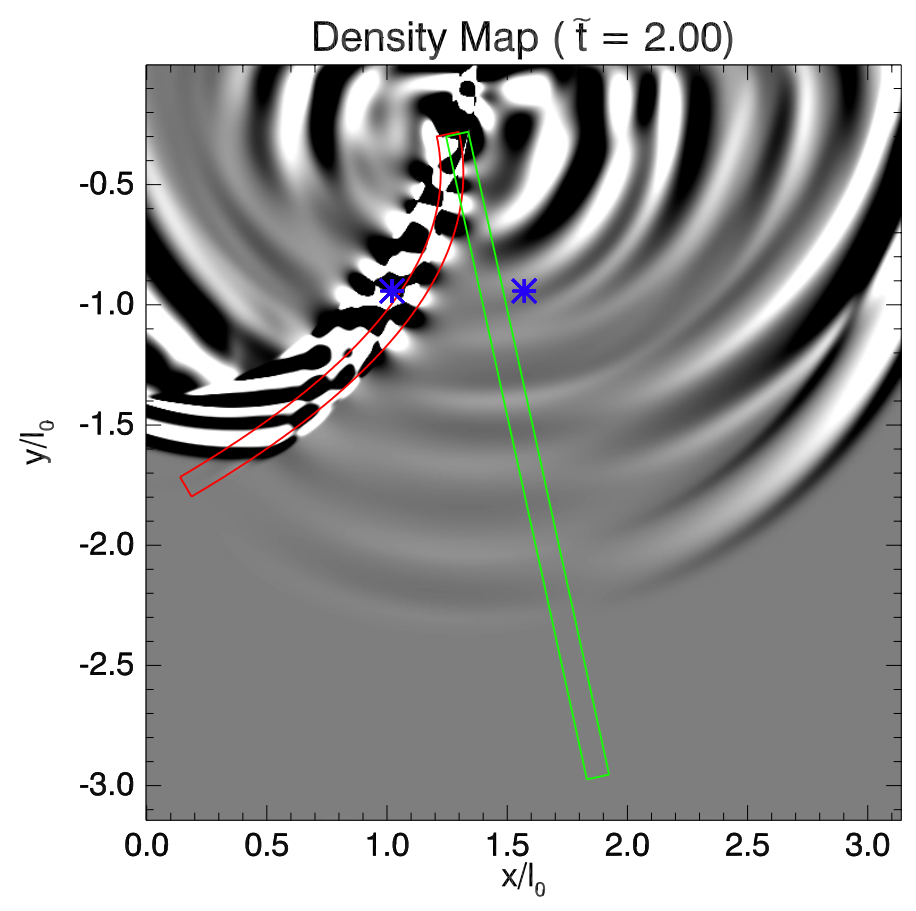

Fig. 6. Running-difference perturbations of the plasma density in the numerical simulation at $\tilde{t}=2$. The red and green lines are the slits used to construct the time-distance maps. The asteriskes show the spatial locations where temporal signals were measured. The FOV shown in this figure covers the right half of the panels shown in Fig. 5. A movie of the numerical simulation is available online.

nature of the signals at both locations is evident. The leaky signal, outside the loop, is more regular. The longer-period spectral components are seen to arrive at the observational point earlier than the shorter-period components. A similar behaviour is seen for the wave train guided by the loop, while the signal is less regular. The reason for the irregularity is that the signal at the point of observation is a superposition of several guided signals. The period of the signal varies in time, but its order of magnitude estimate can be obtained with the use of Eq. (4) of Roberts et al. (1984). The period $P$, the external Alfvén speed $C_{\text {Ae }}$, which is approximately the phase speed of the wave train, and the width $a$ of the wave guide can be combined in a dimensionless parameter $P C_{\mathrm{Ae}} / a$ that is of the order of unity. The period of the signal is about 0.25 normalised time units. Assuming that the external Alfvén speed is 1 normalised speed unit, and the width of the wave guide at the observational point is about 0.25 normalised spatial units, we conclude that the results obtained are consistent with this estimation.

Note that the same process also applies to the upward propagating part of the wave train later on, that is, after it passes the loop apex, but we do not consider these times in this study. For a coronal loop with smaller expansion than the potential field model we used, a height-dependent cut-off may also be provided by a stratified Alfvén speed; a higher Alfvén speed lower in the corona causes the wavelength of the downwardpropagating wave train to increase and thereby assists leakage. Our chosen equilibrium (see Fig. 4) prevents leakage due to wave tunnelling, which has been studied for curved loops, for example by Brady \& Arber (2005), Verwichte et al. (2006a,b), Díaz et al. (2006), and Díaz (2006). Indeed, the tunnelling effect occurs when the Alfvén speed decreases with distance from the oscillating loop, which does not take place in the considered equilibrium. Our equilibrium is more similar to that of
Van Doorsselaere et al. (2004), that is, to regions of constant Alfvén speed, although sausage modes do not experience the resonant absorption that these authors considered (and neither do kink modes in chosen geometry). The rate of energy release from the loop depends upon the details of the structuring, such as the density-contrast ratio and loop expansion/stratification, and thus has potential application as a seismological tool. The dispersive evolution also depends upon the initial spectral profile of the source (e.g. Nakariakov et al. 2005) and requires a driver that is sufficiently broadband, which can be a short impulsive source that sufficiently localised in space just right.

We would also like to point out that the initial perturbation given by Eq. (3) also excites slow and entropy modes (Murawski et al. 2011). However, in this study we focus on the time interval associated with the development of fast magnetoacoustic waves.

\section{Discussion and conclusions}

To compare the observational results presented in Sect. 2 with the results of numerical modelling described in Sect. 3, we applied the TD map technique to the numerical simulation results in the same way as for the observational data. As in the analysis of observations, we considered two slits: the first is a curved path (the red line in Fig. 6) along the closed-loop structure, and the second is a straight line (green) in an open-field region. The slits are superimposed on numerical density RDIs (Fig. 6) and TD maps are extracted from them.

Both observational and numerical TD maps are shown in Fig. 8. The wave train fronts in observations were determined by eye-inspection in TD maps, taking a series of data points (red dots in the left panel of Fig. 8), along the boundaries of black and bright patches. These points are distributed almost along a straight line, whose slope approximates the average phase speed of the QFP wave trains. The series of data points were fitted with a linear function of the form

$d=d_{0}+v t$,

with $(t, d)$ the variable pair as measured in TD maps, and $d_{0}$ and $v$ the constants determined by least-square fitting. The fitting was performed with the inverted relation of Eq. (4) to consider the time as a dependent variable and minimise errors along it. Indeed, choosing a point in TD maps implies an error both in time and space. By assuming an error of $2 \mathrm{Mm}$ and $12 \mathrm{~s}$ (equivalent to the AIA cadence) for each measurement, spatial and temporal scales for the wave front of $150 \mathrm{Mm}$ and $2.5 \mathrm{~min}(150 \mathrm{~s})$, the relative errors for each variable will be $\Delta d / d \sim 1.33 \%$, and $\Delta t / t \sim 8 \%$, so that the time error affects the estimate of the phase speed to a greater extent. The best-fit lines are over-plotted in TD maps as dashed green lines.

The typical speed (see Table 1) ranges between 900-1200 $\mathrm{km} \mathrm{s}^{-1}$ and the relative errors for the fittings are lower than $10 \%$. This estimate is related to the plane of sky. If we assume that the wave train paths are perpendicular to the solar surface and the underlying AR is at $\theta=28 \mathrm{deg}$ from the solar limb, the speed will be slightly higher. The wave train period $P$ is estimated as the lag between two consecutive wave fronts and is between $0.80-1.50 \mathrm{~min}$. Furthermore, the wavelength of the wave train, that is, the distance between two consecutive fronts estimated as $L=v P$, is $60-110 \mathrm{Mm}$.

The TD maps of the numerical simulation agree well with the observations. According to the normalisation quantities used for the speed and the time, as given in Sect. 3, our numerical simulation runtime corresponds to a physical time of $T_{\text {sim }}=5.33 \mathrm{~min}$ 
G. Nisticò et al.: Observation of a high-quality quasi-periodic rapidly propagating wave train using SDO/AIA
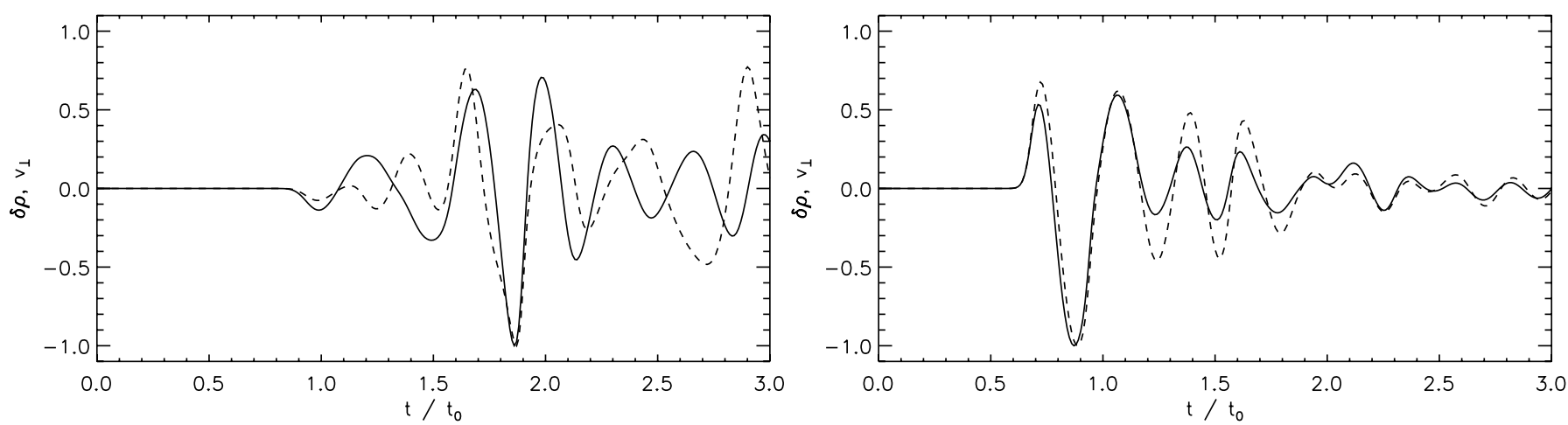

Fig. 7. Time signatures of the density perturbation (solid lines) and the transverse component of the plasma velocity (dashed lines) at points inside (left panel) and outside (right panel) of the wave-guiding coronal loop. The perturbations are normalised to their highest values.
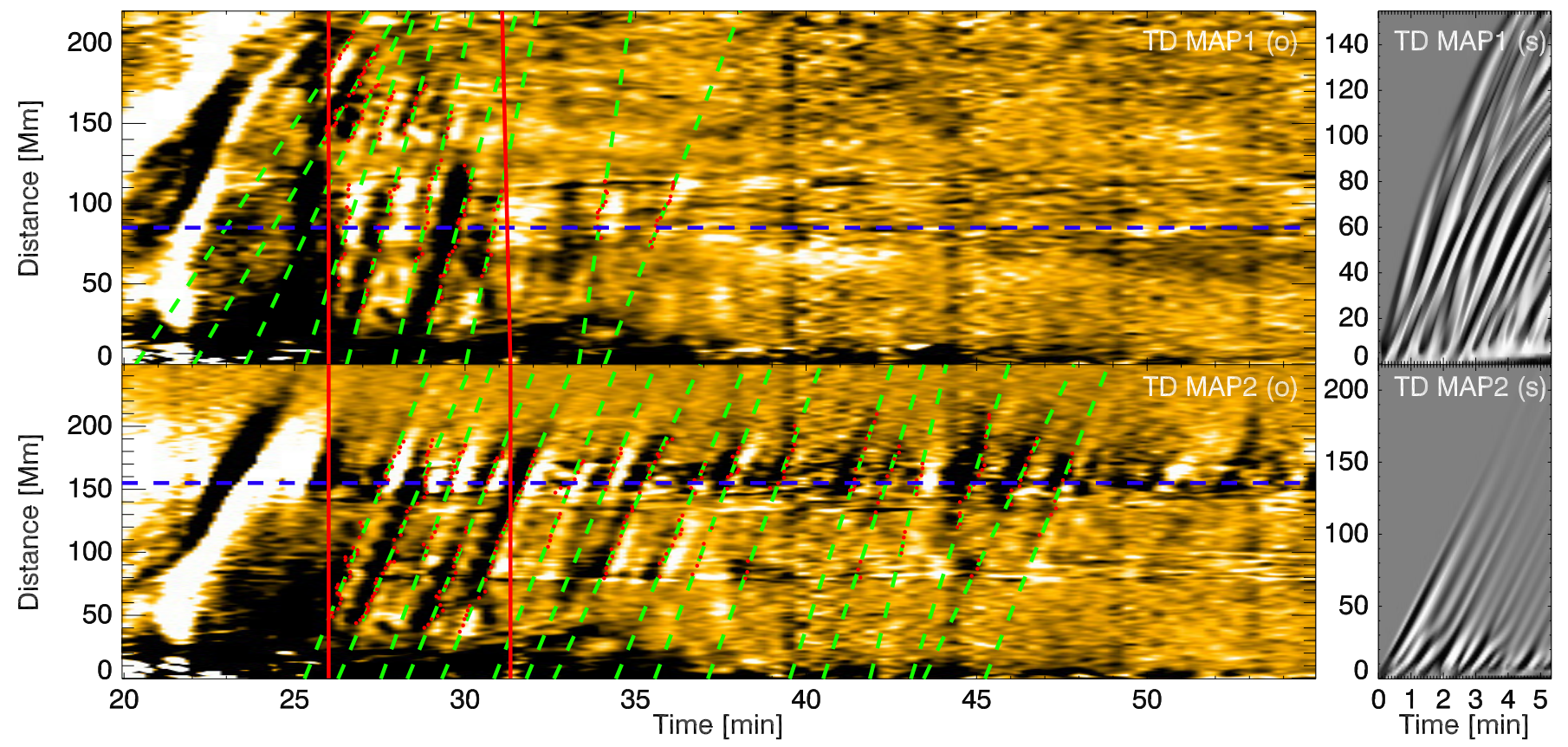

Fig. 8. Time-distance maps from the observations (left) and from the numerical simulation (right). The red dots are the data points that indicate the position of the wave fronts, fitted by the green lines. The vertical continuous red lines delimit a temporal window of about $5 \mathrm{~min}$ from the flare peak for comparison with the numerical simulation. Intensity time series are extracted from the maps at position marked by the dashed blue lines, which are plotted in Fig. 9.

(320 s), taken for example from the flare peak in the observation (vertical red lines in TD MAP1-2 (o)). The general pattern of the QFP wave train in the synthetic TD maps, along the curved (TD MAP1 (s)) and the straight line (TD MAP2 (s)) agrees with our observations, showing more inhomogeneities in the wave train patterns in the first TD map than in the second one, whose slit lies in a predominantly open-field region.

Figure 9 shows the time signals taken at two locations, one in the wave-guiding coronal loop, the other outside it. Wave trains of the EUV intensity perturbations are clearly seen in the signals. Wavelet spectra have the "crazy tadpole" signatures typical for the dispersively evolving guided fast wave trains (Nakariakov et al. 2004). The same information can be obtained in the time domain: longer-period spectral components are clearly seen to arrive at the observational points before the shorter-period spectral components, in full agreement with the theory (see Fig. 7). In addition, for the period $P \approx 60 \mathrm{~s}$, the apparent phase speed is $v \approx 1 \mathrm{Mm} \mathrm{s}^{-1}$ and the observed transverse width of the wave train is $a \approx 20-60 \mathrm{Mm}$, the dimensionless combination $P v / a$ is of the order of unity. This estimate is consistent with the results of numerical modelling.

Previous works on QFP wave trains (Liu et al. 2011; Shen et al. 2013; Shen \& Liu 2012; Yuan et al. 2013) showed an intimate connection between the formation and propagation of QFP wave trains and the time variability of the flaring energy release. In particular, of interest are quasi-periodic pulsations (QPP) in the flare light curve. This can be generally explained as quasi-periodic magnetic reconnection that periodically releases energy that excites wave trains and causes QPP (see Nakariakov \& Melnikov 2009, for a recent review). The flare discussed in this paper shows some quasi-periodic intensity pulsations, especially at the end of the QFP wave train evolution at $171 \AA$. The relationship between QPP and QFP wave train will be the subject of future work. On the other hand, we showed that our numerical modelling of an impulsive energy release demonstrates that dispersive evolution due to transverse structuring (such as a dense coronal loop) can readily generate quasi-periodic propagating fast wave trains with a high signal quality from a single impulsive source. This effect explains the appearance of the 

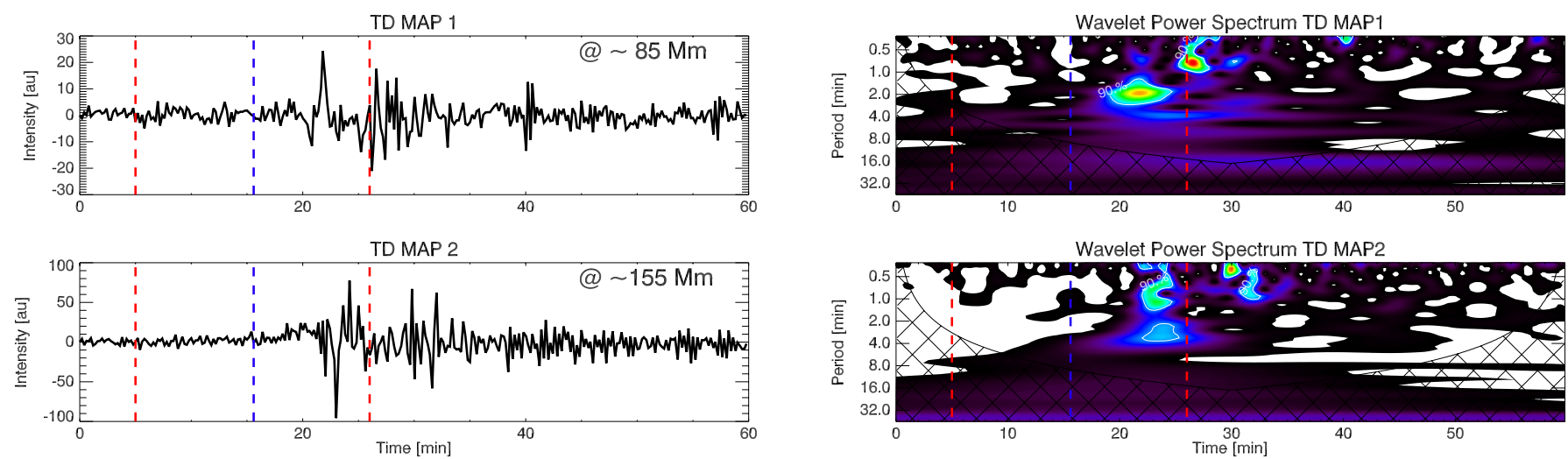

Fig. 9. Left panels: time variation of the EUV intensity perturbations in the rapidly propagating quasi-periodic wave trains, extracted from TD MAP 1 and 2 at a position of about 85 and $155 \mathrm{Mm}$ (see Fig. 8). The perturbations are shown in arbitrary units. The red vertical dashed lines mark the start and peak of the flare. The blue vertical dashed line indicates the time of the sudden increase in the soft X-ray flux. The sequence of the propagating wave train fronts is seen as the increase in the amplitude of the intensity variation. Right panels: wavelet spectra of the signals.

quasi-periodic fast wave trains without the need for a periodic driver. Thus, QFP wave trains may potentially be exploited as a seismological tool for the remote diagnostic of plasma parameters.

Acknowledgements. The data used are courtesy of the SDO/AIA team. This work is supported by the STFC Warwick Astrophysics Consolidated Grant (GN), the European Research Council under the SeismoSun Research Project No. 321141 (DJP, VMN), the BK21 plus program through the National Research Foundation funded by the Ministry of Education of Korea (VMN), and the Russian Foundation of Basic Research under grant 13-02-00044 (VMN).

\section{References}

Anfinogentov, S., Nisticò, G., \& Nakariakov, V. M. 2013, A\&A, 560, A107 Arber, T., Longbottom, A., Gerrard, C., \& Milne, A. 2001, J. Comput. Phys., 171,151

Brady, C. S., \& Arber, T. D. 2005, A\&A, 438, 733

Cooper, F. C., Nakariakov, V. M., \& Williams, D. R. 2003, A\&A, 409, 325

De Moortel, I. 2009, Space Sci. Rev., 149, 65

De Moortel, I. \&, Nakariakov, V. M. 2012, Roy. Soc. London Phil. Trans. Ser. A, 370,3193

Díaz, A. J. 2006, A\&A, 456, 737

Díaz, A. J., Zaqarashvili, T., \& Roberts, B. 2006, A\&A, 455, 709

Edwin, P. M., \& Roberts, B. 1983, Sol. Phys., 88, 179

Jelínek, P., \& Karlický, M. 2012, A\&A, 537, A46

Jelínek, P., Karlický, M., \& Murawski, K. 2012, A\&A, 546, A49

Karlický, M., Jelínek, P., \& Mészárosová, H. 2011, A\&A, 529, A96

Karlický, M., Mészárosová, H., \& Jelínek, P. 2013, A\&A, 550, A1

Katsiyannis, A. C., Williams, D. R., McAteer, R. T. J., et al. 2003, A\&A, 406, 709

Liu, W., Title, A. M., Zhao, J., et al. 2011, ApJ, 736, L13
Liu, W., Ofman, L., Nitta, N. V., et al. 2012, ApJ, 753, 52

Mészárosová, H., Karlický, M., Rybák, J., \& Jiřička, K. 2009a, ApJ, 697, L108 Mészárosová, H., Sawant, H. S., Cecatto, J. R., et al. 2009b, Adv. Space Res., 43, 1479

Mészárosová, H., Karlický, M., \& Rybák, J. 2011, Sol. Phys., 273, 393

Mészárosová, H., Dudík, J., Karlický, M., Madsen, F. R. H., \& Sawant, H. S. 2013, Sol. Phys., 283, 473

Murawski, K., \& Roberts, B. 1993a, Sol. Phys., 143, 89

Murawski, K., \& Roberts, B. 1993b, Sol. Phys., 144, 255

Murawski, K., Zaqarashvili, T. V., \& Nakariakov, V. M. 2011, A\&A, 533, A18

Nakariakov, V. M., \& Melnikov, V. F. 2009, Space Sci. Rev., 149, 119

Nakariakov, V. M., \& Roberts, B. 1995, Sol. Phys., 159, 399

Nakariakov, V. M., Arber, T. D., Ault, C. E., et al. 2004, MNRAS, 349, 705

Nakariakov, V. M., Pascoe, D. J., \& Arber, T. D. 2005, Space Sci. Rev., 121, 115

Nakariakov, V. M., Hornsey, C., \& Melnikov, V. F. 2012, ApJ, 761, 134

Nisticò, G., Nakariakov, V. M., \& Verwichte, E. 2013, A\&A, 552, A57

Ofman, L., Liu, W., Title, A., \& Aschwanden, M. 2011, ApJ, 740, L33

Pascoe, D. J., Nakariakov, V. M., \& Arber, T. D. 2007, A\&A, 461, 1149

Pascoe, D. J., Nakariakov, V. M., \& Kupriyanova, E. G. 2013, A\&A, 560, A97

Patsourakos, S., Vourlidas, A., Wang, Y. M., Stenborg, G., \& Thernisien, A 2009, Sol. Phys., 259, 49

Roberts, B. 1981a, Sol. Phys., 69, 39

Roberts, B. 1981b, Sol. Phys., 69, 27

Roberts, B., Edwin, P. M., \& Benz, A. O. 1983, Nature, 305, 688

Roberts, B., Edwin, P. M., \& Benz, A. O. 1984, ApJ, 279, 857

Shen, Y., \& Liu, Y. 2012, ApJ, 753, 53

Shen, Y.-D., Liu, Y., Su, J.-T., et al. 2013, Sol. Phys., 288, 585

Sych, R., Zaqarashvili, T. V., Nakariakov, V. M., et al. 2012, A\&A, 539, A23

Van Doorsselaere, T., Debosscher, A., Andries, J., \& Poedts, S. 2004, A\&A, 424, 1065

Verwichte, E., Foullon, C., \& Nakariakov, V. M. 2006a, A\&A, 449, 769

Verwichte, E., Foullon, C., \& Nakariakov, V. M. 2006b, A\&A, 452, 615

Wang, T. 2011, Space Sci. Rev., 158, 397

Yuan, D., Shen, Y., Liu, Y., et al. 2013, A\&A, 554, A144

Zajtsev, V. V. \& Stepanov, A. V. 1975, Issledovaniia Geomagnetizmu Aeronomii, i Fizike Solntsa, 37, 3 